\title{
A spatially resolved study of the synchrotron emission and titanium in Tycho's supernova remnant using NuSTAR
}

Lopez, Laura A.; Grefenstette, Brian W.; Reynolds, Stephen P.; An, Hongjun; Boggs, Steven E.; Christensen, Finn Erland; Craig, William W.; Eriksen, Kristoffer A.; Fryer, Chris L.; Hailey, Charles J. Total number of authors: 15

Published in:

Astrophysical Journal

Link to article, DOI:

10.1088/0004-637X/814/2/132

Publication date:

2015

Document Version

Publisher's PDF, also known as Version of record

Link back to DTU Orbit

Citation (APA):

Lopez, L. A., Grefenstette, B. W., Reynolds, S. P., An, H., Boggs, S. E., Christensen, F. E., Craig, W. W., Eriksen, K. A., Fryer, C. L., Hailey, C. J., Harrison, F. A., Madsen, K. K., Stern, D. K., Zhang, W. W., \& Zoglauer, A. (2015). A spatially resolved study of the synchrotron emission and titanium in Tycho's supernova remnant using NuSTAR. Astrophysical Journal, 814(2), [132]. https://doi.org/10.1088/0004-637X/814/2/132

\section{General rights}

Copyright and moral rights for the publications made accessible in the public portal are retained by the authors and/or other copyright owners and it is a condition of accessing publications that users recognise and abide by the legal requirements associated with these rights.

- Users may download and print one copy of any publication from the public portal for the purpose of private study or research.

- You may not further distribute the material or use it for any profit-making activity or commercial gain

- You may freely distribute the URL identifying the publication in the public portal 


\title{
A SPATIALLY RESOLVED STUDY OF THE SYNCHROTRON EMISSION AND TITANIUM IN TYCHO'S SUPERNOVA REMNANT USING NUSTAR
}

\author{
Laura A. Lopez ${ }^{1}$, Brian W. Grefenstette ${ }^{2}$, Stephen P. Reynolds ${ }^{3}$, Honguun An $^{4}$, Steven E. Boggs ${ }^{5}$, \\ Finn E. Christensen ${ }^{6}$, William W. Craig ${ }^{5}$, Kristoffer A. Eriksen ${ }^{7}$, Chris L. Fryer ${ }^{7}$, Charles J. Hailey ${ }^{8}$, \\ Fiona A. Harrison ${ }^{2}$, Kristin K. Madden ${ }^{2}$, Daniel K. Stern ${ }^{9}$, William W. Zhang ${ }^{10}$, and Andreas Zoglauer ${ }^{5}$ \\ ${ }^{1}$ Department of Astronomy and Center for Cosmology \& Astro-Particle Physics, The Ohio State University, Columbus, OH 43210, USA; lopez.513@osu.edu \\ ${ }^{2}$ Cahill Center for Astrophysics, 1216 E. California Blvd., California Institute of Technology, Pasadena, CA 91125, USA \\ 3 Physics Department, NC State University, Raleigh, NC 27695, USA \\ ${ }_{5}^{4}$ Department of Physics, McGill University, Montreal, Quebec, H3A 2T8, Canada \\ ${ }^{5}$ Space Sciences Laboratory, University of California, Berkeley, CA 94720, USA \\ ${ }^{6}$ DTU Space, National Space Institute, Technical University of Denmark, Elektrovej 327, DK-2800 Lyngby, Denmark \\ ${ }^{7}$ CCS-2, Los Alamos National Laboratory, Los Alamos, NM 87545, USA \\ ${ }^{8}$ Columbia Astrophysics Laboratory, Columbia University, New York, NY 10027, USA \\ ${ }^{9}$ Jet Propulsion Laboratory, California Institute of Technology, Pasadena, CA 91109, USA \\ ${ }^{10}$ NASA Goddard Space Flight Center, Greenbelt, MD 20771, USA \\ Received 2015 April 26; accepted 2015 October 11; published 2015 November 30
}

\begin{abstract}
We report results from deep observations ( $\sim 750 \mathrm{ks})$ of Tycho's supernova remnant (SNR) with NuSTAR. Using these data, we produce narrow-band images over several energy bands to identify the regions producing the hardest $\mathrm{X}$-rays and to search for radioactive decay line emission from ${ }^{44} \mathrm{Ti}$. We find that the hardest $(>10 \mathrm{keV}) \mathrm{X}$-rays are concentrated in the southwest of Tycho, where recent Chandra observations have revealed high emissivity "stripes" associated with particles accelerated to the knee of the cosmic-ray spectrum. We do not find evidence of ${ }^{44} \mathrm{Ti}$, and we set limits on its presence and distribution within the SNR. These limits correspond to an upper-limit ${ }^{44} \mathrm{Ti}$ mass of $M_{44}<2.4 \times 10^{-4} M_{\odot}$ for a distance of $2.3 \mathrm{kpc}$. We perform a spatially resolved spectroscopic analysis of 66 regions across Tycho. We map the best-fit rolloff frequency of the hard X-ray spectra, and we compare these results to measurements of the shock expansion and ambient density. We find that the highest energy electrons are accelerated at the lowest densities and in the fastest shocks, with a steep dependence of the rolloff frequency with shock velocity. Such a dependence is predicted by models where the maximum energy of accelerated electrons is limited by the age of the SNR rather than by synchrotron losses, but this scenario requires far lower magnetic field strengths than those derived from observations in Tycho. One way to reconcile these discrepant findings is through shock obliquity effects, and future observational work is necessary to explore the role of obliquity in the particle acceleration process.
\end{abstract}

Key words: ISM: individual objects: (Tycho's SNR) - ISM: supernova remnants - X-rays: ISM

\section{INTRODUCTION}

Tycho's supernova remnant (SNR G120.1+1.4; hereafter Tycho) is widely believed to be the remnant of an SN Ia explosion observed in 1572 (Stephenson \& Green 2002; Badenes et al. 2006; Krause et al. 2008; Rest et al. 2008). Evidence of particle acceleration in Tycho was found first by Pravdo \& Smith (1979), who detected non-thermal X-ray emission up to $25 \mathrm{keV}$ with HEAO-1. Initial Chandra X-ray Observatory images spatially resolved the non-thermal X-ray emission and showed that it originates from narrow filaments around the rim of Tycho (Hwang et al. 2002; Bamba et al. 2005; Warren et al. 2005). Such features are now thought to be common among young SNRs (see Reynolds 2008 for a review). A deep Chandra program revealed several nonthermal, high emissivity "stripes" in the projected interior of the SNR, a result that was interpreted as direct evidence of particles accelerated to the "knee" of the cosmic-ray (CR) spectrum at around $3 \mathrm{PeV}$ (Eriksen et al. 2011, though see Bykov et al. 2011 for an alternative interpretation). Recently, Tycho has been detected in $\mathrm{GeV} \gamma$-rays with Fermi (Giordano et al. 2012) and in $\mathrm{TeV} \gamma$-rays with VERITAS (Acciari et al. 2011). The $\gamma$-ray spectrum is consistent with diffusiveshock acceleration (DSA) either of CR protons (Berezhko et al. 2013) or of two lepton populations (Atoyan \& Dermer 2012).

Recent X-ray studies of Tycho have reported the possible detection of radioactive decay lines of ${ }^{44} \mathrm{Ti}$ (Troja et al. 2014; Wang \& $\mathrm{Li} 2014$ ) and of Ti-K line emission at $\sim 4.9 \mathrm{keV}$ (Miceli et al. 2015). As the properties of the ${ }^{44} \mathrm{Ti}$ (like yield, spatial distribution, and velocities) in young SNRs directly probe the underlying explosion mechanism of the supernova (see, e.g., Magkotsios et al. 2010), constraints on these parameters are useful to motivate and test simulations. The ${ }^{44} \mathrm{Ti}$ isotope has a half-life of 60 years (Ahmad et al. 2006) and originates from $\alpha$-rich freeze-out near nuclear statistical equilibrium (e.g., Timmes et al. 1996; Woosley et al. 2002). Radioactive decay lines of ${ }^{44} \mathrm{Ti}$ to ${ }^{44} \mathrm{Sc}$ and then to ${ }^{44} \mathrm{Ca}$ are observable at $\sim 68,78$, and $1157 \mathrm{keV}$, and these features have been detected in young core-collapse SNRs Cassiopeia A (e.g., Iyudin et al. 1994; Vink et al. 2001; Renaud et al. 2006b; Grefenstette et al. 2014) and SN 1987A (Grebenev et al. 2012; Boggs et al. 2015). ${ }^{44} \mathrm{Ti}$ can also manifest itself through a $4.1 \mathrm{keV}$ line, which occurs from the filling of the inner-shell vacancy resulting from the decay of ${ }^{44} \mathrm{Ti}$ to ${ }^{44} \mathrm{Sc}$ by electron capture. A feature at this energy has been detected in Chandra X-ray spectra of the young SNR G1.9+0.3 (Borkowski et al. 2010). To date, no Type Ia SNRs have definitive 
Table 1

NuSTAR Observation Log

\begin{tabular}{lrr}
\hline \hline ObsID & Exposure & UT Start Date \\
\hline 40020001002 & $339 \mathrm{ks}$ & 2014 Apr 12 \\
40020011002 & $147 \mathrm{ks}$ & 2014 May 31 \\
40020001004 & $262 \mathrm{ks}$ & 2014 Jul 18 \\
\hline
\end{tabular}

detections of the 68,78 , or $1157 \mathrm{keV}$ lines associated with ${ }^{44} \mathrm{Ti}$ (e.g., Dupraz et al. 1997; Iyudin et al. 1999; Renaud et al. 2006a; Zoglauer et al. 2015).

In this paper, we present hard X-ray images and spatially resolved spectra from a set of NUSTAR observations of Tycho totaling $\sim 750 \mathrm{ks}$. Launched in 2012, NuSTAR is the first satellite to focus at hard X-ray energies of 3-79 keV (Harrison et al. 2013). The primary scientific motivation of our observing program was to map and characterize the non-thermal X-ray emission in Tycho and to detect or constrain the spatial distribution of ${ }^{44} \mathrm{Ti}$. The paper is outlined as follows. In Section 2, we describe the data reduction and analysis procedures. In Section 3.1, we present the composite narrowband NUSTAR images of the hard X-ray emission in Tycho, and in Section 3.2, we exploit NuSTAR images to set upper limits on the presence of ${ }^{44} \mathrm{Ti}$. In Section 3.3, we report the results from a systematic spatially resolved spectroscopic analysis across the SNR. In Section 4, we discuss the implications of our results, specifically related to the particle acceleration properties of Tycho (in Section 4.2) and to ${ }^{44} \mathrm{Ti}$ searches in young SNRs (in Section 4.1). We summarize our conclusions in Section 5.

\section{OBSERVATIONS AND DATA ANALYSIS}

Tycho was observed by NuSTAR three times from 2014 April to July, as listed in Table 1, with a total net integration of $748 \mathrm{ks}$. We reduced these data using NuSTAR Data Analysis Software (NuSTARDAS) Version 1.3.1 and NuSTAR CALDB Version 20131223. We performed the standard pipeline data processing with nupipeline, with the stricter criteria for the passages through the South Atlantic Anomaly (SAA) and the "tentacle"-like region near the SAA to reduce background uncertainties.

Using the resulting cleaned event files, we produced images of different energy bands using the FTOOL xselect and generated associated exposure maps using nuexpomap. Because Tycho is a bright, extended source, we opted to model the background and produce synthetic, energy-dependent background images for background subtraction. We followed the procedure detailed in Wik et al. (2014) and Grefenstette et al. (2014) to estimate the background components and their spatial distribution. Subsequently, we combined the vignetting- and exposure-corrected FPMA and FPMB images from all epochs using ximage.

The combined images were then deconvolved by the on-axis NuSTAR point-spread function (PSF) using the max_likelihood AstroLib IDL routine. The script employs Lucy-Richardson deconvolution, an iterative procedure to derive the maximum likelihood solution. We set the maximum number of iterations to 50, as more iterations did not lead to any significant changes in the resulting images. We note that this routine assumes that the data can be characterized by a Poisson distribution, but background subtraction causes the images to not follow strictly

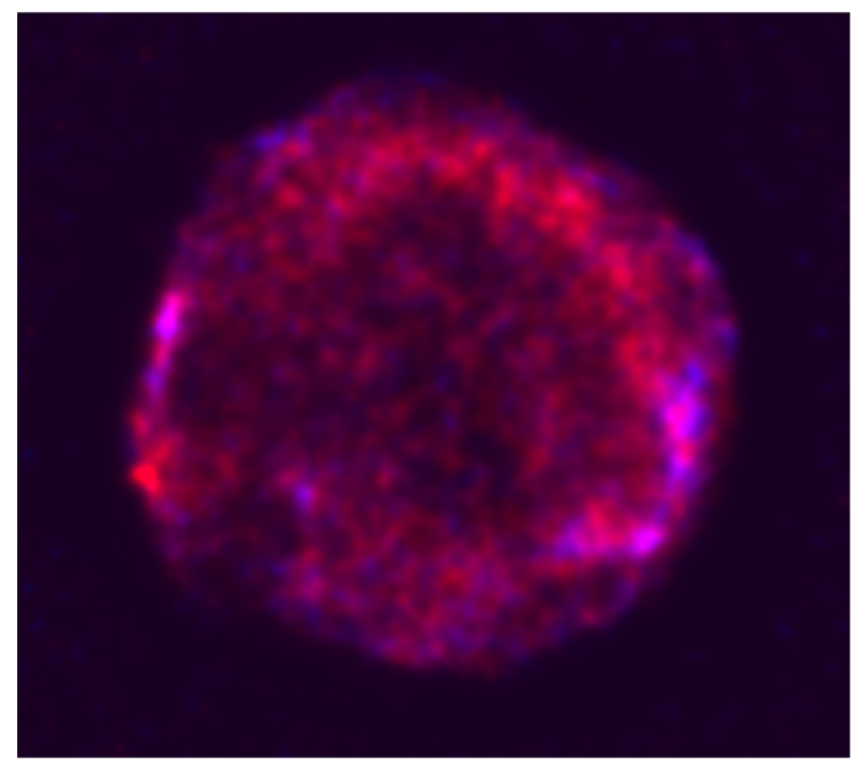

Figure 1. Deconvolved, background-subtracted NUSTAR image from the combined $\sim 750 \mathrm{ks}$ observation of Tycho, where red is $6-7 \mathrm{keV}$ (which is dominated by Fe line emission) and blue is $10-20 \mathrm{keV}$. The SNR is $\sim 9^{\prime}$ in diameter. In this and all subsequent images, north is up and east is left.

a Poisson distribution. Thus, the deconvolved images in Figures 1 and 2 are presented for qualitative purposes only, and we do not use them for any quantitative results.

We performed spatially resolved spectroscopic analyses by extracting and modeling spectra from locations across the SNR. Using the nuproducts FTOOL, we extracted source spectra and produced ancillary response files (ARFs) and redistribution matrix files (RMFs) from each of the three observations and both the $\mathrm{A}$ and $\mathrm{B}$ modules ( 3 ObsIDS $\times 2$ modules $=6$ spectra per region). Furthermore, we utilized the nuskybgd routines (presented in detail in Wik et al. 2014) to simulate the associated background spectra. We employed the addascaspec FTOOL to combine the six source pulse-height amplitude (PHA) files, ARFs, and background PHA files associated with each region. The six RMFs were added using the addrmf FTOOL. As discussed by Grefenstette et al. (2015), the addition of spectra from different observations leads to systematic effects of the order of a few percent in the best-fit normalizations of the spectra.

For comparison to the NUSTAR data, we analyzed archival Chandra and National Radio Astronomy Observatory (NRAO) Very Large Array (VLA) observations of Tycho. The Chandra Advanced CCD Imaging Spectrometer (ACIS) observed Tycho in 2009 April for a total integration of $734 \mathrm{ks}$ (Eriksen et al. 2011). Reprocessed data were downloaded from the Chandra archive (ObsIDs 10093-10097, 10902-10906), and composite exposure-corrected images were produced of the 4-6 keV continuum using the flux_image command in the Chandra Interactive Analysis of Observations (CIAO) software Version 4.6. The VLA observed Tycho at $1.505 \mathrm{GHz}$ on 2001 July 10 in the $\mathrm{C}$ configuration for 174 minutes (program AR464, PI: Reynoso). The reduced image, with a beam size of 15 ! 8 by 13 ". 5 , was downloaded from the NRAO/VLA image archive. $^{11}$

\footnotetext{
11 https://science.nrao.edu/facilities/vla/archive/index
} 

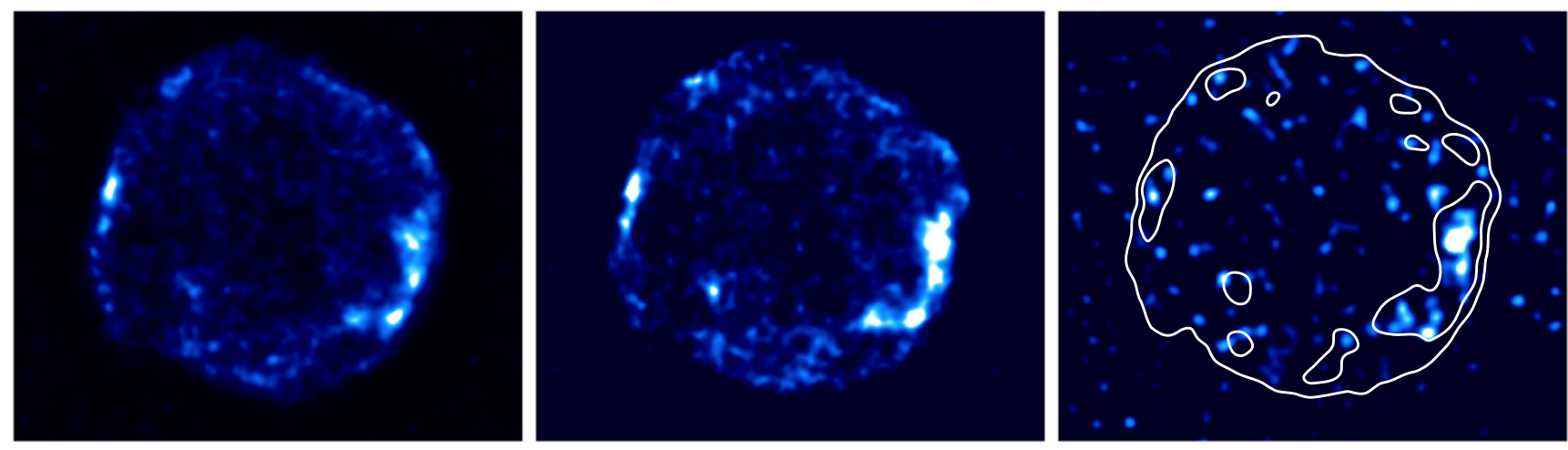

Figure 2. Deconvolved, background-subtracted NuSTAR images of three hard energy bands: 8-10 keV (left), 10-20 keV (middle), and 20-40 keV (right). White contours in the right panel are from the $10-20 \mathrm{keV}$ band to guide the eye.
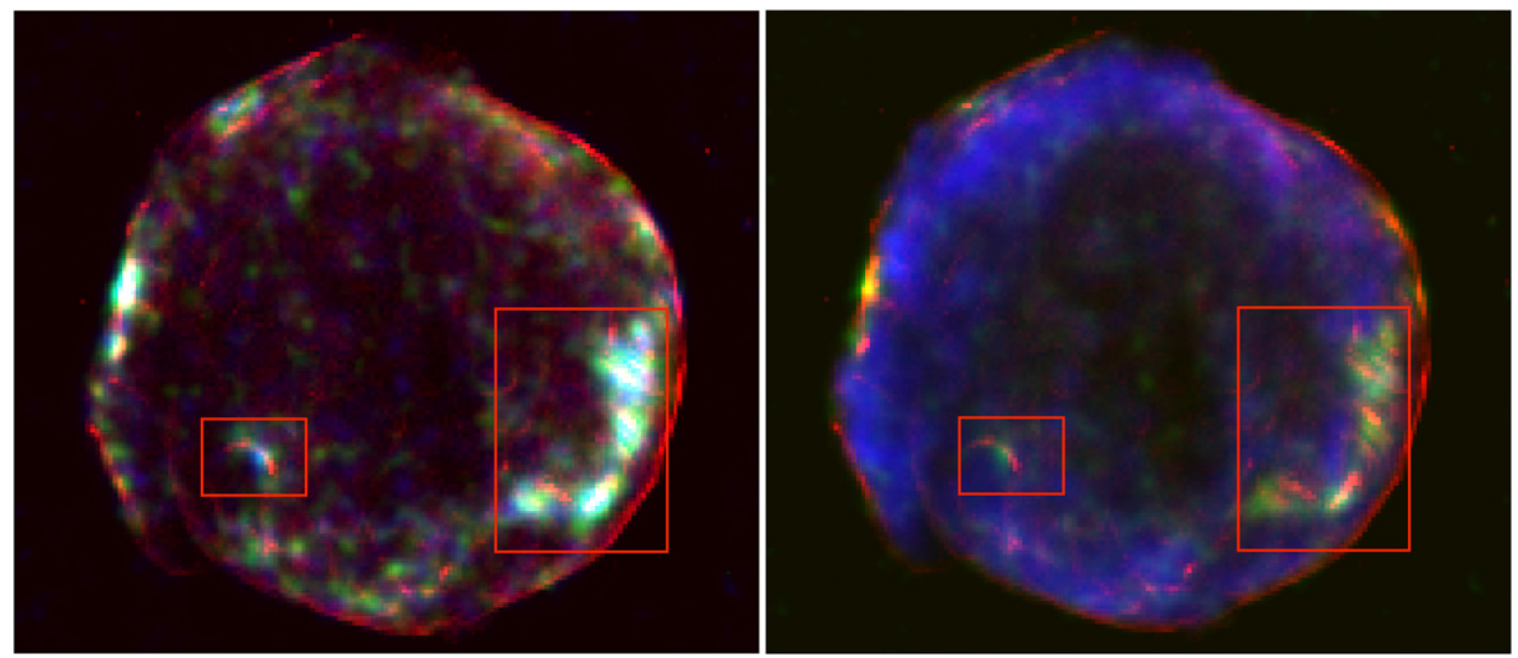

Figure 3. Left: three-color image, with 4-6 keV Chandra data in red (binned by a factor of eight), 8-10 keV deconvolved NuSTAR data in green, and 10-20 keV deconvolved NUSTAR data in blue. The hardest X-rays are brightest in the west of the SNR and coincide with the non-thermal X-ray "stripes" (denoted by the large red rectangle) seen in the deep Chandra data and analyzed by Eriksen et al. (2011). The small rectangle corresponds to the "hook" of non-thermal emission described in the text. Right: three-color image, with 4-6 keV Chandra data in red, 10-20 keV deconvolved NuSTAR data in green, and 20-cm VLA data in blue. We note that the NuSTAR, Chandra, and VLA images have pixel sizes of 2".5, 3!"9, and 4!"2, respectively.

\section{RESULTS}

\subsection{NuSTAR Imaging}

Figures 1 and 2 show the deconvolved, backgroundsubtracted NUSTAR images of Tycho from the combined $\sim 750$ ks observation in several energy bands. NUSTAR detects the "fluffy" Fe-rich ejecta found predominantly in the northwest of Tycho (Decourchelle et al. 2001; Yamaguchi et al. 2014) as well as non-thermal X-rays with up to energies of $\sim 40 \mathrm{keV}$. The hard $(>8 \mathrm{keV}) \mathrm{X}$-rays are predominantly distributed around the rim of the SNR, with the peak at the location of the "stripes" identified in Chandra observations (see Figure 3 for a comparison).

In the right panel of Figure 3, we compare the $20-\mathrm{cm}$ radio morphology seen by the VLA to the 4-6 keV Chandra and $10-20 \mathrm{keV}$ NUSTAR images. The radio emission is distributed in a ring interior to the narrow, non-thermal filaments at the SNR periphery found by Chandra. Generally, the bright, hard $\mathrm{X}$-ray knots detected by NUSTAR do not coincide with the radio emission. Overall, we find that the Chandra $4-6 \mathrm{keV}$ band has a similar morphology as revealed in the NUSTAR images up to $\sim 20 \mathrm{keV}$. The hardest X-rays $(20-40 \mathrm{keV})$ are concentrated around the stripes, as well as near a "hook" in the southwest of the SNR (denoted by the small red rectangle in Figure 3).

We also convolved the Chandra 4-6 keV image with a Gaussian to match the resolution of the NUSTAR data, and the comparison of the result to the $10-20 \mathrm{keV} \mathrm{NuSTAR}$ data is shown in Figure 4. As the $10-20 \mathrm{keV}$ emission is almost exclusively non-thermal, the locations where the $4-6 \mathrm{keV}$ emission is coincident reveals that the latter is substantially non-thermal in nature. The converse scenario (where 4-6 keV emission is found without $10-20 \mathrm{keV}$ emission) indicates that the softer band may be dominated by thermal emission. We find that the 4-6 keV emission is largely coincident with the harder band, except in the northwest, where previous X-ray studies have found significant emission from the ejecta (Decourchelle et al. 2001; Yamaguchi et al. 2014). Thus, we conclude that the 4-6 keV Chandra band is likely dominated by non-thermal emission across Tycho, except in the northwest.

In Figure 5, we compare the NuSTAR 20-40 keV emission to the $\mathrm{GeV}$ and $\mathrm{TeV}$ centroids reported by Giordano et al. (2012) and Acciari et al. (2011), respectively. The GeV localization (from Fermi observations with a 95\% confidence level) coincides with the hard X-rays from the "hook" feature in the 


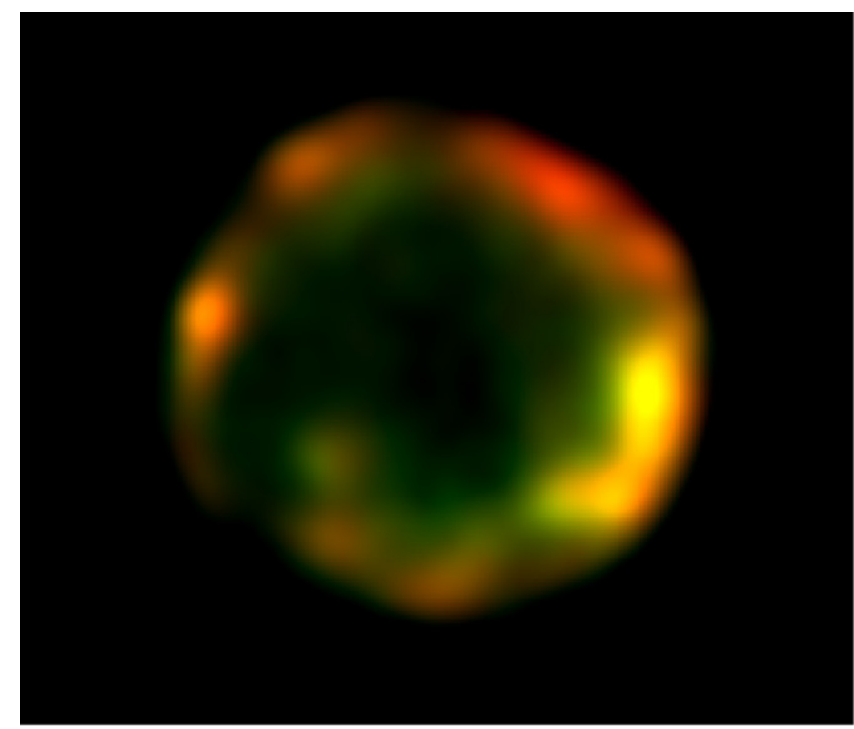

Figure 4. Two-color image of Tycho to demonstrate the spatial coincidence of the 4-6 keV Chandra data (in red) and the 10-20 keV deconvolved NuSTAR data (in green). We have convolved the Chandra image with a Gaussian function to match the resolution of the NUSTAR image. As the emission in the $10-20 \mathrm{keV}$ band is almost exclusively non-thermal, locations where the 4-6 keV emission is coincident indicates that the latter is also non-thermal in nature.

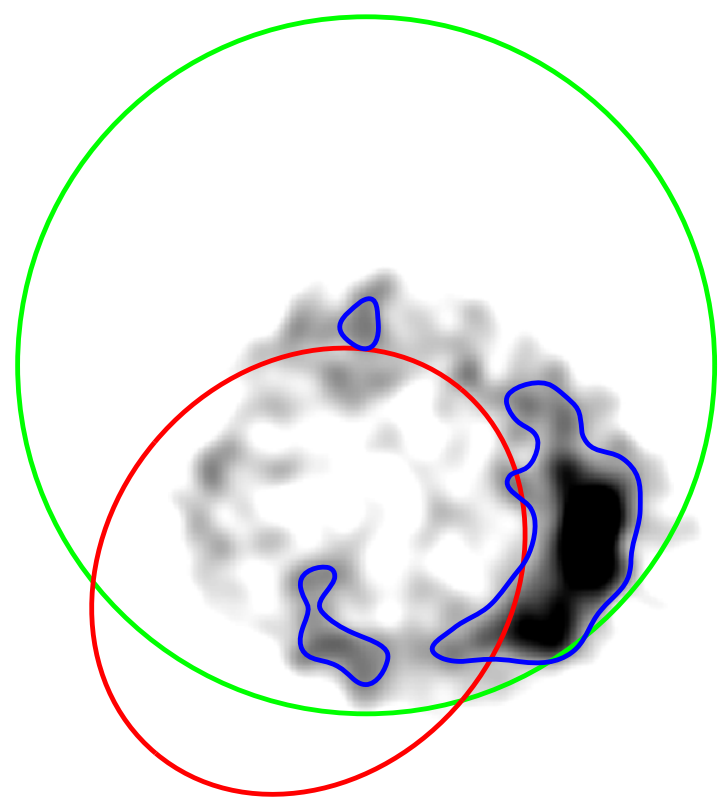

Figure 5. Comparison of the high-energy detections of Tycho, with the Fermi $95 \%$ confidence error circle denoted in red (for photons of energy $E>1 \mathrm{GeV}$; from Giordano et al. 2012), the VERITAS error circle in green (for energies $E>1 \mathrm{TeV}$; from Acciari et al. 2011), and the NUSTAR 20-40 keV band contours in blue. The image is the backgroundsubtracted NUSTAR 20-40 keV image smoothed with a Gaussian function of width $\sigma=5$ pixels.

southeast of the SNR, although we note that the "stripe" region is just outside the $95 \%$ error circle. The $\mathrm{TeV}$ detection is consistent with a point source at the $0.11 \approx 6$ ! 6 PSF of VERITAS, which is of the same order as the angular extent $\left(\sim 8^{\prime}\right)$ of the SNR. Thus, it is not possible with the currently reported VERITAS observations to determine the origin of the $\mathrm{TeV}$ emission.

\subsection{Upper Limits on ${ }^{44} \mathrm{Ti}$}

We do not find any point-like or extended emission in the remnant using the $65-70 \mathrm{keV}$ band containing the $68 \mathrm{keV}{ }^{44} \mathrm{Ti}$ emission line. We searched using the method from Grefenstette et al. (2014), where we compared the observed image to a simulated background image. We searched on multiple spatial scales by convolving both the NUSTAR image and the simulated image with a top hat function, varying the radius of the function from 3 to $15 \mathrm{NUSTAR}$ pixels (roughly 7.5-30 arcsec). We computed the Poisson probability that the observed counts in each pixel could have been produced by the predicted background flux, where a low probability indicates that the pixel likely contains a source count. We do not find any evidence for excess emission on any spatial scales.

We place upper limits on the diffuse extended emission in Tycho arising from the $68 \mathrm{keV}$ line. We extracted spectra and produced an ARF and RMF for source regions with radii from 1 to 5 arcmin centered on the remnant. For example, in Figure 6, we give the spectra and background model of the FPMA and FPMB data for a $4^{\prime}$ radius extraction region. For each radius, we modeled the source using a combination of a power-law continuum with two Gaussian lines of fixed relative intensity centered at 68 and $78 \mathrm{keV}$ and fit the data over the 10-79 keV bandpass. We do not find any evidence for line emission in any of the source regions.

Our upper limits are dependent on the intrinsic width of the ${ }^{44} \mathrm{Ti}$ lines. We computed the $3 \sigma$ confidence limits on the line normalizations for fixed line widths of $0.1,1$, or $3 \mathrm{keV}$, which correspond to velocities of $\approx 733,7330$, and $22,000 \mathrm{~km} \mathrm{~s}^{-1}$, respectively. The resulting $3 \sigma$ upper limits as a function of radius are shown in Figure 7 . In all cases, the lower limits are zero.

To effectively compare these NUSTAR upper limits with results from other missions, we convert all previous measurements and limits to epoch 2014. In Table 2, we list these values for observations with the Compton Gamma-ray Observatory/ COMPTEL, INTEGRAL/IBIS, and Swift/BAT. To obtain epoch 2014 fluxes (which are also given in Table 2), we adopt a half-life of 58.9 years (Ahmad et al. 2006). The NuSTAR upper limits may be used in conjunction with the previous results to assess the likely spatial distribution of the ${ }^{44} \mathrm{Ti}$. In particular, our upper limits for radii $\lesssim 2^{\prime}$ are less than the reported Swift/BAT detection (at epoch 2014) of $(1.2 \pm 0.6) \times$ $10^{-5} \mathrm{ph} \mathrm{cm}^{-2} \mathrm{~s}^{-1}$, suggesting that the ${ }^{44} \mathrm{Ti}$ is distributed over a larger radius of Tycho.

Recently, Miceli et al. (2015) reported detection of the Ti-K line complex at $\sim 4.9 \mathrm{keV}$ using stacked XMM-Newton spectra from Tycho. In particular, these authors identified the Ti feature in two regions of enhanced emission from Fe-group elements, in the northwest and in the southeast of the SNR (regions \#1 and \#3 in Figure 2 of Miceli et al. 2015). Motivated by this result, we searched for the $68 \mathrm{keV}$ line at these locations in the NUSTAR data (the region files were provided graciously by M. Miceli). We did not detect any signal above the background from either region, with lower limits of zero in all cases. The $3 \sigma$ confidence flux upper limits are $3.9 \times 10^{-6} \mathrm{ph} \mathrm{cm}^{-2} \mathrm{~s}^{-1}$ and $7.3 \times 10^{-7} \mathrm{ph} \mathrm{cm}^{-2} \mathrm{~s}^{-1}$ for regions $\# 1$ and $\# 3$, respectively, assuming a line width of $1 \mathrm{keV}$. 

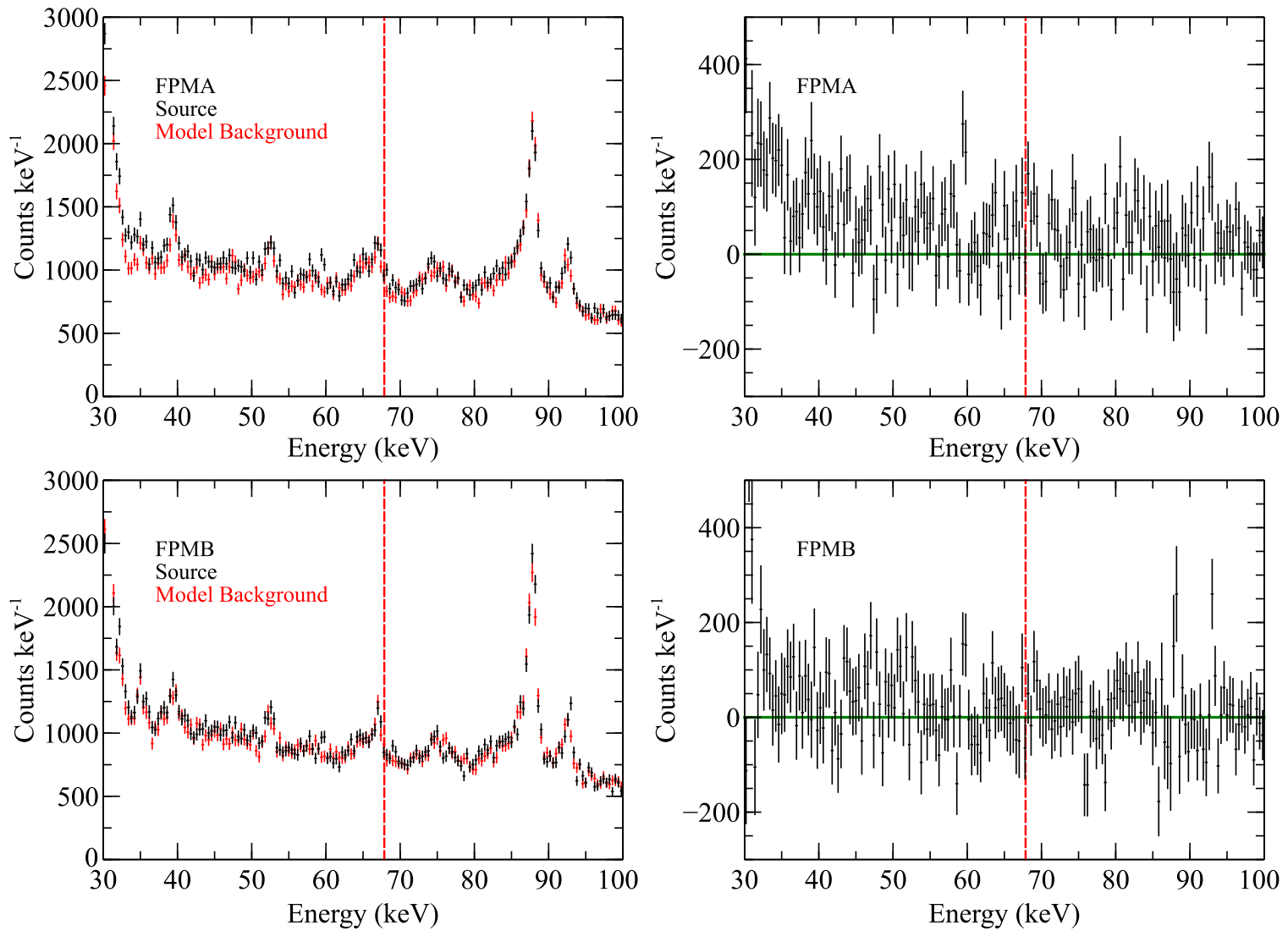

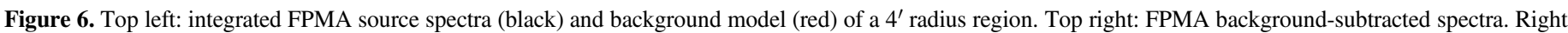

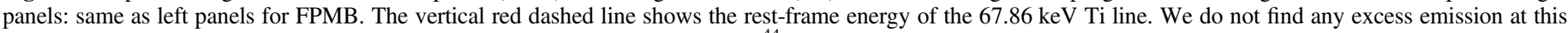
line energy relative to the background model, and thus we set upper limits on ${ }^{44} \mathrm{Ti}$.

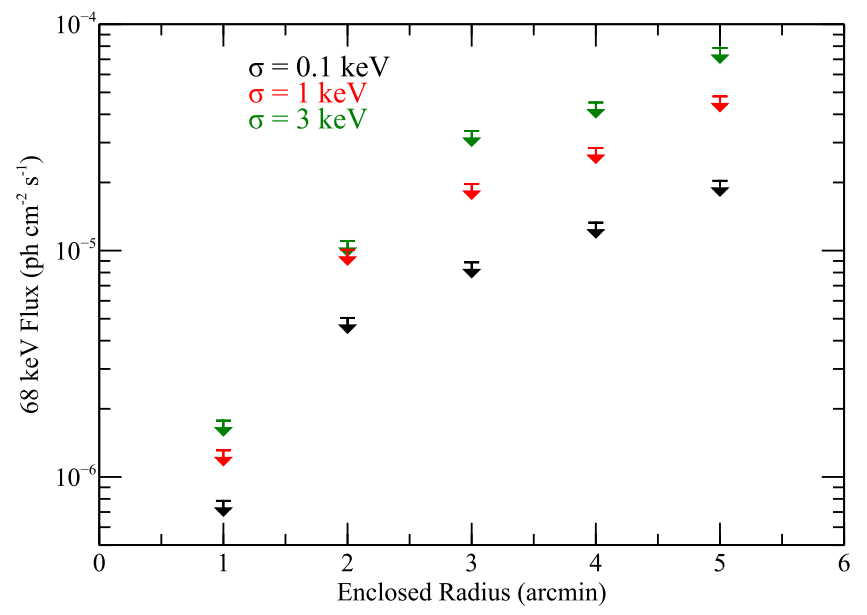

Figure 7. Black, red, and green points show the $3 \sigma$ confidence limits as a function of radius for line widths $\sigma$ of $0.1,1$, and $3 \mathrm{keV}$ (corresponding to velocities of $\sim 730,7300$, and 22,000 $\mathrm{km} \mathrm{s}^{-1}$ ).

\subsection{Spatially Resolved Spectroscopy with NuSTAR}

We performed spatially resolved spectroscopic analyses by extracting and modeling spectra from sixty-six $1^{\prime}$ by $1^{\prime}$ boxes across the SNR (see Figure 8). To evaluate the non-thermal emission, we fit the spectra in the $10-50 \mathrm{keV}$ range with an absorbed srcut model. In our fits, we have fixed the absorbing column to $N_{\mathrm{H}}=7 \times 10^{21} \mathrm{~cm}^{-2}$ (Eriksen et al. 2011), and we have grouped the spectra to a minimum of 30 counts bin' ${ }^{-1}$.
The srcut model describes the spectrum as that radiated by a power-law energy distribution of electrons with an exponential cutoff at energy $E_{\max }$ (Reynolds \& Keohane 1999). That spectrum cuts off roughly as $\exp \left(-\sqrt{\nu / \nu_{\text {rolloff }}}\right)$ (though the fitting uses the complete expression). The rolloff photon energy $E_{\text {rolloff }}=h \nu_{\text {rolloff }}$ is related to the maximum energy of the accelerated electrons $E_{\max }$ by

$$
E_{\max }=120\left(\frac{h \nu_{\text {rolloff }}}{1 \mathrm{keV}}\right)^{1 / 2}\left(\frac{B}{\mu \mathrm{G}}\right)^{-1 / 2} \mathrm{TeV},
$$

where $B$ is the magnetic field strength and we have corrected for a small numerical error in Reynolds \& Keohane (1999).

In XSPEC, the srcut model is characterized by three parameters: the rolloff frequency $\nu_{\text {rolloff }}$, the mean radio-to-Xray spectral index $\alpha$, and the $1 \mathrm{GHz}$ radio flux density $F_{1 \mathrm{GHz}}$. To limit the free parameters in the fit, we estimated $F_{1 \mathrm{GHz}}$ in each of the sixty-six regions by measuring the $1.505 \mathrm{GHz}$ flux density in the NRAO/VLA data assuming a radio spectral index of $\alpha=-0.65$ (Kothes et al. 2006). The derived flux densities at $1 \mathrm{GHz}$ using this procedure are listed in Table 3. We note that without additional single-dish observations, the interferometric VLA data are missing flux from the largest scales. Based on previous radio studies of Tycho, we estimate the missing flux is of the order of $\sim 10 \%$ of the values listed in Table 3.

Table 3 lists the spectroscopic results, giving the best-fit rolloff frequency $\nu_{\text {rolloff }}$ and the $\chi^{2}$ and degrees of freedom (dof) for all sixty-six regions. We find that all of the regions are 
Table 2

Previous Measurements and Limits ${ }^{\mathrm{a}}$ on ${ }^{44} \mathrm{Ti}$

\begin{tabular}{lcccc}
\hline \hline Instruments & $\begin{array}{c}\text { Years of } \\
\text { Obs. }\end{array}$ & $\begin{array}{c}\text { Mean Year } \\
\text { of Obs. }\end{array}$ & $\begin{array}{c}\text { Flux at Mean Year } \\
\left(\times 10^{-5} \mathrm{ph} \mathrm{cm}^{-2} \mathrm{~s}^{-1}\right)\end{array}$ & $\begin{array}{c}\text { Flux at Epoch 2014 } \\
\left(\times 10^{-5} \mathrm{ph} \mathrm{cm}^{-2} \mathrm{~s}^{-1}\right)\end{array}$ \\
\hline CGRO/COMPTEL & $1991-1997$ & 1994 & $<1.6$ & $<1.3$ \\
INTEGRAL/IBIS & $2002-2006$ & 2004 & $<1.5$ & $<1.4$ \\
Swift/BAT & $2004-2013$ & 2008.5 & $1.3 \pm 0.6$ & $1.2 \pm 0.6$ \\
\hline
\end{tabular}

Note.

${ }^{\text {a }}$ CGRO/COMPTEL upper limit is $2 \sigma$, from Dupraz et al. (1997) and Iyudin et al. (1999); the INTEGRAL/IBIS upper limit is $3 \sigma$, from Renaud et al. (2006a) and Wang \& Li (2014); the Swift/BAT detection is the $90 \%$ confidence limit, from Troja et al. (2014).

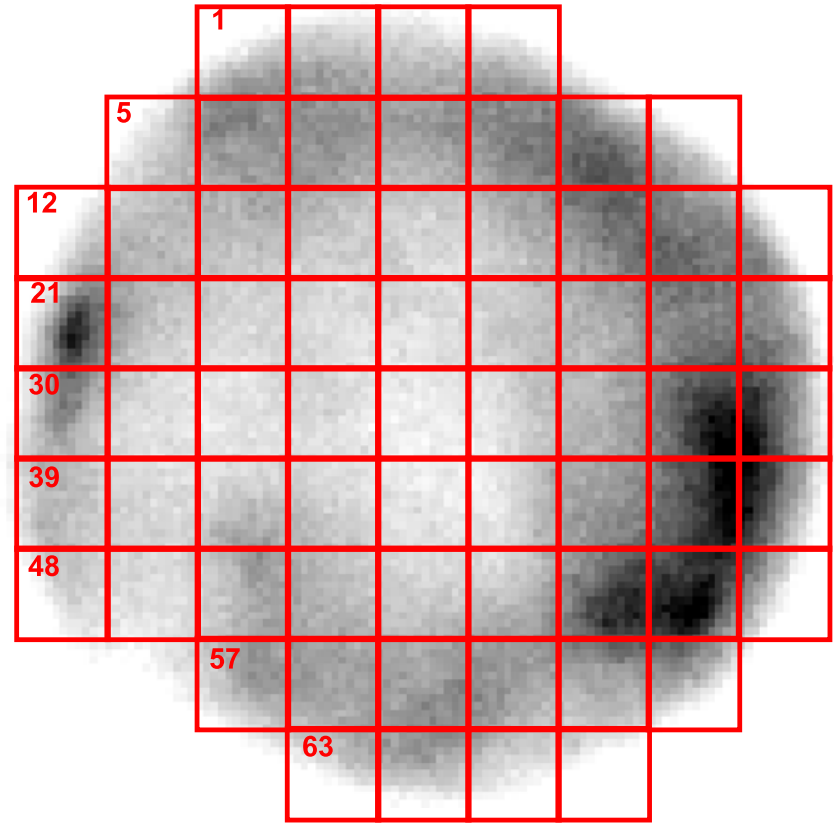

Figure 8. Background-subtracted, merged 3-20 keV image of Tycho with $1^{\prime}$ by $1^{\prime}$ boxes overplotted denoting the regions where NUSTAR spectra were extracted and modeled.

fit well by the srcut models, with $\chi^{2} /$ dof $=\chi_{\mathrm{r}}^{2} \approx 0.82-1.26$ with $\sim 100-200$ dof per region. Figure 9 shows example spectra from six of the regions, and Figure 10 maps the best-fit rolloff frequency $\nu_{\text {rolloff values across Tycho. We find }}$ substantial variation of a factor of five in $\nu_{\text {rolloff }}$. The largest values of $\nu_{\text {rolloff }} \gtrsim 3 \times 10^{17} \mathrm{~Hz}$ (corresponding to a rolloff energy of $E_{\text {rolloff }}=1.24 \mathrm{keV}$ ) are concentrated in the west of the SNR where Eriksen et al. (2011) identified the "stripes" in Tycho. Furthermore, we find elevated rolloff frequencies in the northeast of the SNR, with $\nu_{\text {rolloff }} \approx 2 \times 10^{17} \mathrm{~Hz}$ (corresponding to $E_{\text {rolloff }} \approx 0.83 \mathrm{keV}$ ), where the SNR is thought to be interacting with molecular material (Reynoso et al. 1999; Lee et al. 2004). The lowest values of $\nu_{\text {rolloff }} \sim 10^{17} \mathrm{~Hz}$ (corresponding to $E_{\text {rolloff }} \approx 0.42 \mathrm{keV}$ ) are found in the southeast and north of the SNR. For the range of $E_{\text {rolloff }} \approx 0.4-2.0 \mathrm{keV}$, we find in our spatially resolved spectroscopic analyses, Equation $(1)$ gives a maximum energy $E_{\max } \approx(75-170)(B / \mu \mathrm{G})^{-1 /}$ ${ }^{2} \mathrm{TeV}$. Assuming a magnetic field $B=200 \mu \mathrm{G}$ across Tycho (Parizot et al. 2006), this relation gives $E_{\max }=5-12 \mathrm{TeV}$.

Previous X-ray studies of Tycho have also measured $\nu_{\text {rolloff }}$ over the entire SNR or along prominent synchrotron filaments. Using ASCA data from Tycho, Reynolds \& Keohane (1999) found $\nu_{\text {rolloff }} \approx 8.8 \times 10^{16} \mathrm{~Hz}$, and using the first Chandra observation of Tycho, Hwang et al. (2002) derived even lower $\nu_{\text {rolloff }}$ toward the northeast, northwest, and southwest rim, with $\nu_{\text {rolloff }} \sim 3-7 \times 10^{16} \mathrm{~Hz}$. More recently, in their analysis of the deep Chandra observations of Tycho, Eriksen et al. (2011) fit absorbed srcut models to the hard "stripes" and a series of filaments projected in the south of Tycho (corresponding to our regions \#51 and 52) which they called the "faint ensemble." They found that the stripes have $\nu_{\text {rolloff }} \sim 2 \times 10^{18} \mathrm{~Hz}$ while the faint ensemble had softer spectra, with $\nu_{\text {rolloff }} \approx$ $3 \times 10^{17} \mathrm{~Hz}$.

Our NUSTAR results confirm that the stripes have harder emission than other regions in Tycho, but our $90 \%$ confidence limits exclude $\nu_{\text {rolloff }} \gtrsim 5 \times 10^{17} \mathrm{~Hz}$ there. The softest regions (with $\nu_{\text {rolloff }} \approx 10^{17} \mathrm{~Hz}$ ) are comparable to the values found by Reynolds \& Keohane (1999) integrated over Tycho. The discrepancy between our $90 \%$ confidence limits and the hard stripes analyzed by Eriksen et al. (2011) may be due to two issues. First, the angular resolution of NUSTAR is not sufficient to resolve the individual stripes, and thus our spectra appear softer as they are averaging over larger areas of the SNR. Second, Eriksen et al. (2011) fit the Chandra data from 4.2 to $10 \mathrm{keV}$, and these spectra may not have had reliable leverage to model the high-energy rolloff. Additionally, we note that the effective area of Chandra drops precipitously above $8 \mathrm{keV}$.

We note that we have assumed a constant radio spectral index of $\alpha=-0.65$, the value derived by Kothes et al. (2006) based on the integrated flux densities at 408 and $1420 \mathrm{MHz}$. However, the radio spectrum is actually concave, and thus $\alpha$ may flatten at higher frequencies (Reynolds \& Ellison 1992). If we instead adopted $\alpha=-0.5$ in our analysis, the best-fit values of $\nu_{\text {rolloff }}$ change substantially, decreasing by about a factor of five. However, the assumption of $\alpha=-0.5$ is not favored statistically based on the $\chi^{2}$ computed from those fits. For example, region \#21 has $\chi^{2}=235$ (147) with 160 dof in the $\alpha=-0.5(-0.65)$ case. Additionally, the $\alpha=-0.5$ fits dramatically over-predict the continuum flux below $10 \mathrm{keV}$, whereas the $\alpha=-0.65$ fits adequately describe the continuum down to $3 \mathrm{keV}$ (see Figure 9). Generally, regardless of the choice of $\alpha$, the qualitative trend in the spatial distribution of $\nu_{\text {rolloff }}$ (e.g., with greater $\nu_{\text {rolloff }}$ in the west of the SNR) remains the same.

\section{DISCUSSION}

\subsection{The Detection of ${ }^{44} \mathrm{Ti}$ in Young SNRs}

The yield, spatial distribution, and velocity distribution of ${ }^{44} \mathrm{Ti}$ in a young SNR are a direct probe of the underlying explosion mechanism of the originating supernova (see, e.g., Magkotsios et al. 2010). Among core-collapse SNRs, Cassiopeia A remains the only Galactic source with a confirmed detection of ${ }^{44} \mathrm{Ti}$ (Iyudin et al. 1994; Grefenstette et al. 2014), 
Table 3

Spatially Resolved Spectroscopy Fit Results Using SRCUT ${ }^{\mathrm{a}}$

\begin{tabular}{|c|c|c|c|c|c|c|c|}
\hline Region & $\begin{array}{c}F_{1 \mathrm{GHz}} \\
(\mathrm{Jy})\end{array}$ & $\begin{array}{c}\nu_{\text {rolloff }} \\
\left(\times 10^{17} \mathrm{~Hz}\right)\end{array}$ & $\chi^{2} /$ dof & Region & $\begin{array}{c}F_{1 \mathrm{GHz}} \\
(\mathrm{Jy})\end{array}$ & $\begin{array}{c}\nu_{\text {rolloff }} \\
\left(\times 10^{17} \mathrm{~Hz}\right)\end{array}$ & $\chi^{2} / \mathrm{dof}$ \\
\hline 1 & 0.55 & $1.91 \pm 0.06$ & $109 / 126$ & 34 & 0.42 & $1.55 \pm 0.05$ & $117 / 110$ \\
\hline 2 & 1.32 & $1.17 \pm 0.03$ & $166 / 138$ & 35 & 0.53 & $1.59 \pm 0.05$ & $138 / 123$ \\
\hline 3 & 1.29 & $1.08 \pm 0.03$ & $130 / 122$ & 36 & 0.75 & $1.63 \pm 0.04$ & $133 / 147$ \\
\hline 4 & 0.89 & $1.22 \pm 0.04$ & $116 / 117$ & 37 & 1.18 & $2.25 \pm 0.04$ & $247 / 213$ \\
\hline 5 & 0.97 & $1.33 \pm 0.04$ & $129 / 128$ & 38 & 0.58 & $3.43 \pm 0.08$ & $204 / 186$ \\
\hline 6 & 1.85 & $1.17 \pm 0.02$ & $156 / 158$ & 39 & 1.38 & $1.11 \pm 0.03$ & $161 / 132$ \\
\hline 7 & 1.51 & $1.21 \pm 0.02$ & $161 / 154$ & 40 & 0.90 & $1.20 \pm 0.03$ & $135 / 121$ \\
\hline 8 & 0.97 & $1.40 \pm 0.03$ & $163 / 140$ & 41 & 0.74 & $1.66 \pm 0.04$ & $164 / 142$ \\
\hline 9 & 1.27 & $1.30 \pm 0.03$ & $152 / 148$ & 42 & 0.76 & $1.41 \pm 0.03$ & $133 / 127$ \\
\hline 10 & 1.39 & $1.33 \pm 0.03$ & $144 / 143$ & 43 & 0.46 & $1.62 \pm 0.05$ & $137 / 114$ \\
\hline 11 & 0.40 & $2.01 \pm 0.08$ & $93 / 108$ & 44 & 0.48 & $1.78 \pm 0.05$ & $132 / 121$ \\
\hline 12 & 0.34 & $2.11 \pm 0.08$ & $134 / 109$ & 45 & 0.85 & $1.78 \pm 0.04$ & $155 / 159$ \\
\hline 13 & 1.51 & $1.09 \pm 0.02$ & $145 / 141$ & 46 & 1.08 & $2.59 \pm 0.04$ & $208 / 218$ \\
\hline 14 & 1.05 & $1.29 \pm 0.03$ & $144 / 135$ & 47 & 0.47 & $3.66 \pm 0.09$ & $191 / 172$ \\
\hline 15 & 0.74 & $1.45 \pm 0.04$ & $130 / 128$ & 48 & 0.87 & $1.22 \pm 0.04$ & $133 / 117$ \\
\hline 16 & 0.55 & $1.65 \pm 0.05$ & $110 / 120$ & 49 & 1.05 & $1.14 \pm 0.03$ & $134 / 123$ \\
\hline 17 & 0.63 & $1.83 \pm 0.05$ & $124 / 134$ & 50 & 1.16 & $1.36 \pm 0.03$ & $154 / 154$ \\
\hline 18 & 1.15 & $1.48 \pm 0.03$ & $162 / 152$ & 51 & 1.03 & $1.34 \pm 0.03$ & $154 / 147$ \\
\hline 19 & 1.20 & $1.56 \pm 0.03$ & $152 / 159$ & 52 & 0.69 & $1.58 \pm 0.04$ & $147 / 135$ \\
\hline 20 & 0.14 & $5.38 \pm 0.27$ & $145 / 115$ & 53 & 0.71 & $1.81 \pm 0.04$ & $140 / 146$ \\
\hline 21 & 0.67 & $2.52 \pm 0.06$ & $147 / 160$ & 54 & 1.01 & $2.25 \pm 0.04$ & $217 / 198$ \\
\hline 22 & 1.38 & $1.13 \pm 0.02$ & $165 / 136$ & 55 & 1.32 & $2.16 \pm 0.03$ & $214 / 205$ \\
\hline 23 & 0.85 & $1.25 \pm 0.03$ & $136 / 120$ & 56 & 0.19 & $4.22 \pm 0.18$ & $110 / 121$ \\
\hline 24 & 0.62 & $1.42 \pm 0.04$ & $117 / 116$ & 57 & 1.15 & $1.33 \pm 0.03$ & $163 / 144$ \\
\hline 25 & 0.54 & $1.47 \pm 0.04$ & $105 / 114$ & 58 & 1.40 & $1.27 \pm 0.02$ & $164 / 158$ \\
\hline 26 & 0.58 & $1.58 \pm 0.04$ & $122 / 125$ & 59 & 1.36 & $1.37 \pm 0.03$ & $157 / 153$ \\
\hline 27 & 0.80 & $1.54 \pm 0.04$ & $120 / 143$ & 60 & 1.08 & $1.57 \pm 0.03$ & $157 / 157$ \\
\hline 28 & 1.21 & $1.58 \pm 0.03$ & $162 / 167$ & 61 & 1.04 & $1.74 \pm 0.04$ & $127 / 155$ \\
\hline 29 & 0.54 & $2.64 \pm 0.07$ & $179 / 153$ & 62 & 0.48 & $2.45 \pm 0.07$ & $149 / 139$ \\
\hline 30 & 1.09 & $1.55 \pm 0.03$ & $188 / 151$ & 63 & 0.49 & $1.71 \pm 0.06$ & $130 / 115$ \\
\hline 31 & 0.97 & $1.18 \pm 0.03$ & $131 / 118$ & 64 & 0.76 & $1.60 \pm 0.04$ & $126 / 133$ \\
\hline 32 & 0.66 & $1.39 \pm 0.04$ & $113 / 117$ & 65 & 0.53 & $1.69 \pm 0.05$ & $127 / 117$ \\
\hline 33 & 0.64 & $1.37 \pm 0.04$ & $135 / 116$ & 66 & 0.09 & $4.63 \pm 0.31$ & $101 / 94$ \\
\hline
\end{tabular}

Note.

${ }^{a}$ Error bars on the rolloff frequency $\nu_{\text {rolloff }}$ represent the $90 \%$ confidence range.

and SN 1987A in the Large Magellanic Cloud also has a robust ${ }^{44} \mathrm{Ti}$ detection (Grebenev et al. 2012; Boggs et al. 2015). To date, the only probable detection of ${ }^{44} \mathrm{Ti}$ in a Type Ia SNR was reported by Troja et al. (2014), who found a $4 \sigma$ level excess above the continuum in the $60-85 \mathrm{keV}$ band in Swift/BAT observations of Tycho. Wang \& Li (2014) also found a marginal $2.6 \sigma$ detection of excess emission in the $60-90 \mathrm{keV}$ band using INTEGRAL/IBIS, although their $3 \sigma$ upper limit is consistent with the previous INTEGRAL studies of Tycho (Renaud et al. 2006a). Other searches for ${ }^{44} \mathrm{Ti}$ in Type Ia SNRs have yielded upper limits, such as the recent work by Zoglauer et al. (2015) using NuSTAR observations of the young SNR $\mathrm{G} 1.9+0.3$.

Based on our NUSTAR analyses, we do not detect ${ }^{44} \mathrm{Ti}$ in Tycho. We looked both for small scale "clumpy" emission as found in Cassiopeia A (Grefenstette et al. 2014) as well as for diffuse emission throughout the SNR. We find upper limits which are more strict than the previous COMPTEL (Dupraz et al. 1997; Iyudin et al. 1999) and INTEGRAL (Renaud et al. 2006a; Wang \& Li 2014) values, particularly if the ${ }^{44} \mathrm{Ti}$ is concentrated in the central $2^{\prime}$ and/or has slow or moderate velocities (i.e., the 0.1 and $1.0 \mathrm{keV}$ line width cases). For example, our $3 \sigma$ limit in the $0.1 \mathrm{keV}(1.0 \mathrm{keV})$ case at an enclosed radius of $2^{\prime}$ is $5.0 \times 10^{-6}\left(1.0 \times 10^{-5}\right) \mathrm{ph} \mathrm{cm}^{-2} \mathrm{~s}^{-1}$. To be consistent with the possible detection of ${ }^{44} \mathrm{Ti}$ with Swift/ BAT (Troja et al. 2014), our results indicate that the ${ }^{44} \mathrm{Ti}$ is expanding at moderate-to-high velocities $\left(\gtrsim 7000 \mathrm{~km} \mathrm{~s}^{-1}\right)$ and/ or is distributed across the majority $\left(z^{\prime}\right)$ of the SNR.

We have also found no ${ }^{44} \mathrm{Ti}$ in the regions where Miceli et al. (2015) reported detection of the Ti-K line complex using XMM-Newton. This $\sim 4.9 \mathrm{keV}$ feature arises from shockheated, stable ${ }^{48} \mathrm{Ti}$ and ${ }^{50} \mathrm{Ti}$. Thus, the lack of a co-located detection of ${ }^{44} \mathrm{Ti}$ may indicate that the radioactive isotope does not trace the Fe-rich ejecta. Alternatively, the divergent results may suggest that the $4.9 \mathrm{keV}$ feature arises from transitions of $\mathrm{Ca}$ XIX and Ca XX rather than from stable Ti. In the future, these interpretations can be tested via deeper NUSTAR observations to reveal the spatial distribution of ${ }^{44} \mathrm{Ti}$, and through highresolution X-ray spectroscopy (with e.g., Astro- $H$ ) which may distinguish between the $\mathrm{Ca}$ and $\mathrm{Ti}$ spectral features.

The upper limit flux that we have measured of the $67.9 \mathrm{keV}$ line can be employed to set an upper limit on the ${ }^{44} \mathrm{Ti}$ yield in the $\mathrm{SN}$ explosion. The ${ }^{44} \mathrm{Ti}$ mass $M_{44}$ is related to the flux $F_{68}$ 

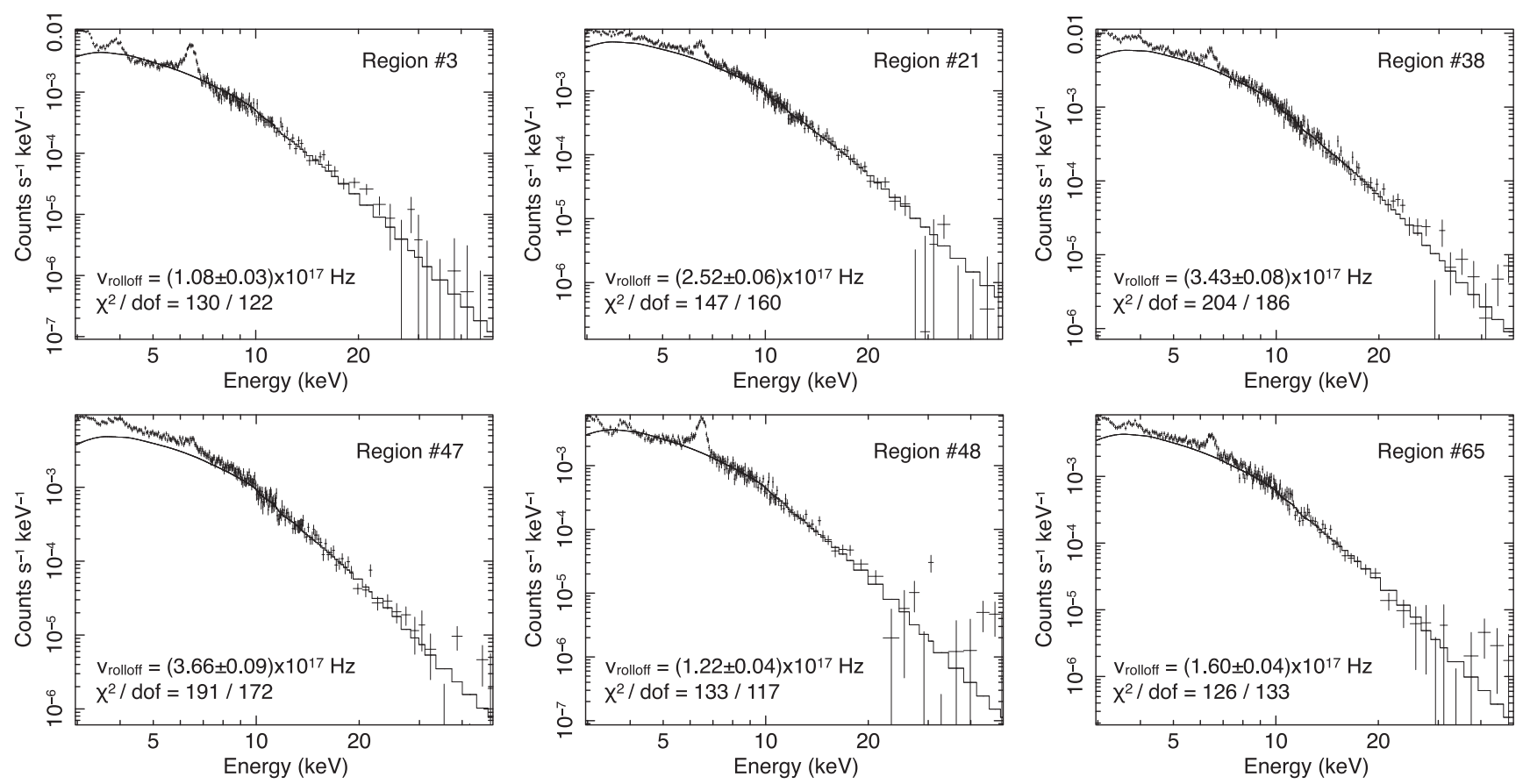

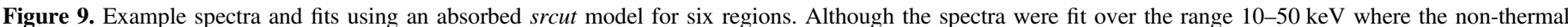

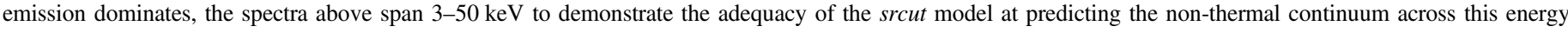
range.

in the $67.9 \mathrm{keV}^{44} \mathrm{Ti}$ line (e.g., Grebenev et al. 2012) by

$$
M_{44}=4 \pi d^{2}\left(44 m_{\mathrm{p}}\right) \tau \exp (t / \tau) F_{68} W^{-1},
$$

where $d$ is the distance to the source, $m_{\mathrm{p}}$ is the mass of a proton, $\tau$ is the mean lifetime of the radioactive titanium (which is related to the half-life $t_{1 / 2}$ by $\tau=t_{1 / 2} / \ln 2 \approx 85$ years for ${ }^{44} \mathrm{Ti}$ : Ahmad et al. 2006), $t$ is the age of the source, and $W$ is the emission efficiency (average numbers of photons per decay) for a given emission line ( $W=0.93$ for the $67.9 \mathrm{keV}{ }^{44} \mathrm{Ti}$ line). Assuming a distance of $D=2.3 \mathrm{kpc}$ and an age of $t=442$ years for Tycho, an upper limit of $F_{68}<2 \times 10^{-5} \mathrm{ph} \mathrm{cm}^{-2} \mathrm{~s}^{-1}$ corresponds to $M_{44}<2.4 \times 10^{-4} M_{\odot}$. Here, we adopted the upper limit $F_{68}$ in the case where the ${ }^{44} \mathrm{Ti}$ is located within a radius of $3^{\prime}$ and has a line width of $1 \mathrm{keV}$, as these most closely match the expected scale and velocity of the shell. For the range of distance estimates in the literature of $D=1.7-5.0 \mathrm{kpc}$ to Tycho, we find upper limits of $M_{44}<1.3 \times 10^{-4} M_{\odot}$ and $M_{44}<10^{-3} M_{\odot}$, respectively.

These upper limits can be compared to the predictions of ${ }^{44} \mathrm{Ti}$ synthesized in SN Ia models. Generally, the amount of ${ }^{44} \mathrm{Ti}$ produced in an SN Ia depends on both the progenitor systems as well as the ignition processes. Simulations predict ${ }^{44} \mathrm{Ti}$ masses ranging from $\sim 10^{-6} M_{\odot}$ in centrally ignited pure deflagration models and $\lesssim 6 \times 10^{-5} M_{\odot}$ for off-center delayed detonation models (e.g., Iwamoto et al. 1999; Maeda et al. 2010). In sub-Chandrasekhar models, greater ${ }^{44} \mathrm{Ti}$ yields are expected, with estimates of $\gtrsim 10^{-3} M_{\odot}$ (e.g., Fink et al. 2010; Woosley \& Kasen 2011; Moll \& Woosley 2013). The mass limits estimated above are most consistent with the models that produce small or moderate yields of ${ }^{44} \mathrm{Ti}$ and disfavor the sub-Chandrasekhar models.

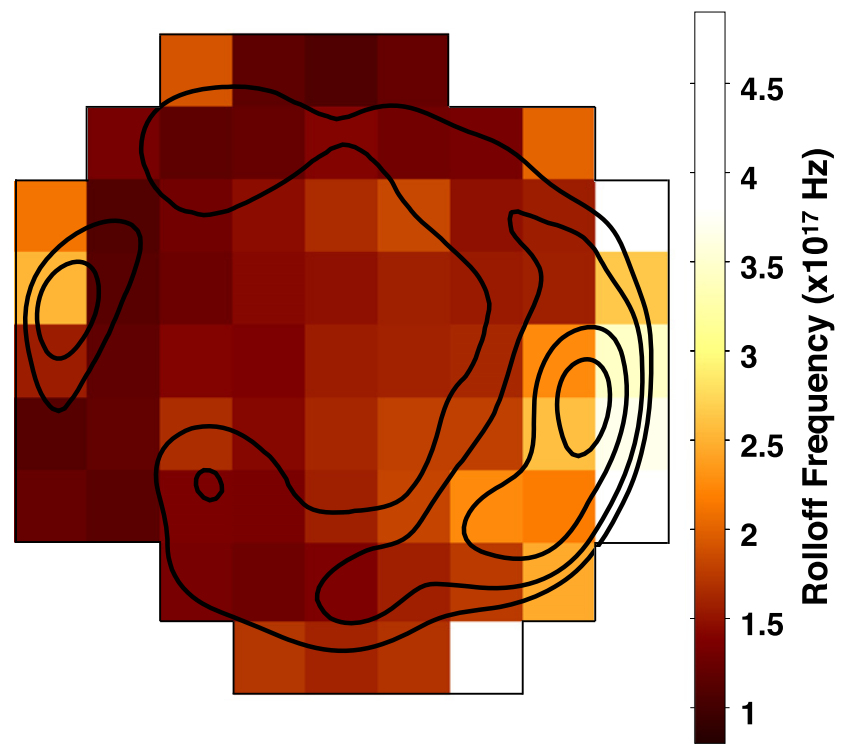

Figure 10. Map of rolloff frequency $\nu_{\text {rolloff }}$ across Tycho, with black contours from the 3-20 keV image in Figure 8. The softest spectra (i.e., those with the smallest best-fit values of $\nu_{\text {rolloff }}$ ) are found in the southeast and north of Tycho, while the hardest spectra are in the northeast and the west. The hardest spectra (with $\nu_{\text {rolloff }} \gtrsim 3 \times 10^{17} \mathrm{~Hz}$ ) are coincident with the "stripes" found in deep Chandra observations of Tycho by Eriksen et al. (2011).

\subsection{Particle Acceleration in Tycho}

To explore the relationship between the non-thermal X-ray emission detected by NuSTAR and shock properties, we compare the spatially resolved spectroscopic results of Section 3.3 to measurements of the shock expansion and the ambient density $n_{\mathrm{o}}$ in Tycho from previous studies.

Expansion measurements of Tycho's rim have been done at radio, optical, and X-ray wavelengths. Using 1375-MHz VLA 


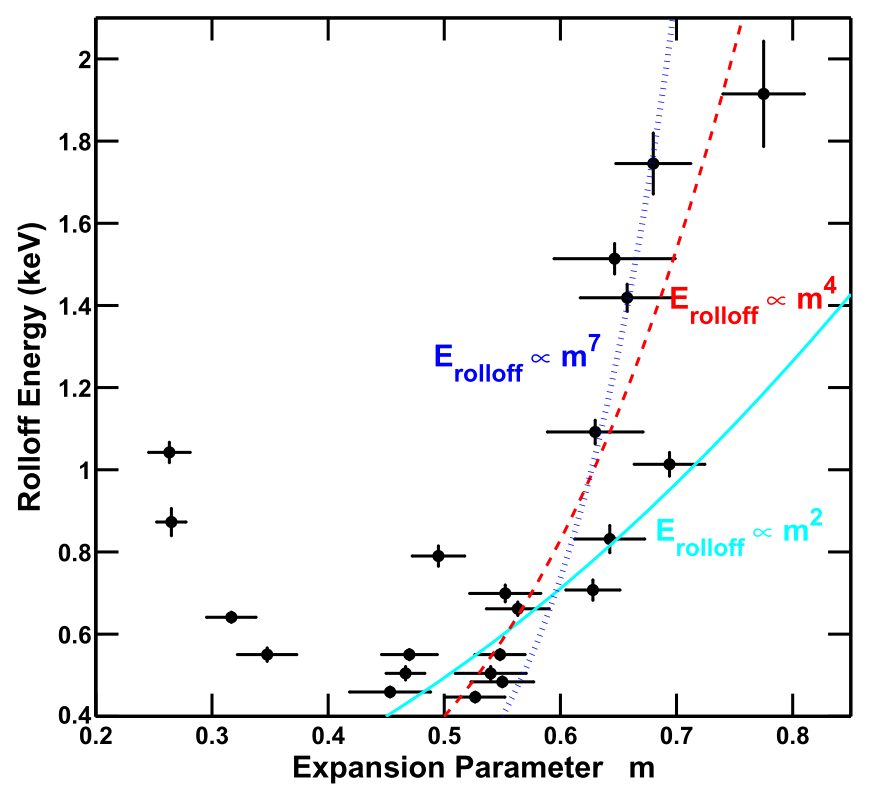

Figure 11. Rolloff energy $E_{\text {rolloff }}$ (determined in Section 3.3) of regions around the rim of Tycho vs. expansion parameter $m$ (as listed in Table 2 of Reynoso et al. 1997). Overplotted are three curves that represent different dependences of $E_{\text {rolloff }}$ on $m: E_{\text {rolloff }} \propto m^{2}$ (cyan solid line), $E_{\text {rolloff }} \propto m^{4}$ (red dashed line), and $E_{\text {rolloff }} \propto m^{7}$ (blue dotted line). These dependences are derived using the expressions in Equations (6)-(8) and given that $R \propto t^{m}$, so $v_{\mathrm{s}}=m R / t \propto m$. The former two trends are those expected if the maximum energy of the electrons is limited by the age of the SNR or by the timescale of the radiative losses, respectively. The latter curve represents the loss-limited case if the B-field strength is not constant (and instead $B \propto v_{\mathrm{s}} \propto m$ ). The $E_{\text {rolloff }} \propto m^{2}$ trend does not match the data for large $m$, and thus, the age-limited scenario is more consistent with our results.

data over a 10-year baseline, Reynoso et al. (1997) measured expansion rates of $\sim 0$ !" $1-0$." $5 \mathrm{yr}^{-1}$, depending on the azimuthal angle. These authors found that the slowest expansion occurs in the east/northeast, where the forward shock is interacting with a dense cloud (Reynoso et al. 1999; Lee et al. 2004), and the fastest expansion is along the western rim. Quantitatively similar results were obtained in the optical by Kamper \& van den Bergh (1978) and in the X-ray by Katsuda et al. (2010). Using the measured expansion index and SNR evolutionary models, Katsuda et al. (2010) inferred a pre-shock ambient density of $n_{\mathrm{o}} \lesssim 0.2 \mathrm{~cm}^{-3}$, with probable local variations of factors of a few based on the substantial differences in the proper motion around the rim.

The shock expansion is described by the radius of the shock with time, $R \propto t^{m}$, where $m$ is the expansion parameter. From this expression, the shock velocity is related to $m$ by $v_{\mathrm{s}}=m R / t$. Thus, the $m$ is a useful indicator of shock velocity, yet it does not require assumptions about the distance to the source. Generally, $m$ is close to unity when the expansion is undecelerated (as in e.g., the free expansion phase of a young SNR). As the shock undergoes deceleration, $m \sim 0.6-0.8$ (Chevalier 1982a, 1982b), and $m=0.4$ once the shock reaches the Sedov phase. Finally, as the shock becomes radiative, $m$ drops below 0.4. While interior pressure is still important, $m=0.33$ ("pressure-driven snowplow"; e.g., Blondin et al. 1998), and then falls to $m=0.25$ when the shock transitions to a pure momentum-conserving stage (Cioffi et al. 1988). In the case of Tycho, Reynoso et al. (1997) demonstrated the expansion parameter $m$ varies around the rim from $m \sim 0.25$ in the east (where the shock is decelerating due to the collision with a dense clump known as knot $g$ : Kamper \& van den Bergh 1978; Ghavamian et al. 2000) up to $m \lesssim 0.8$ in the southwest.

In Figure 11, we show the rolloff energy (found in Section 3.3) versus the expansion parameter $m$ obtained by Reynoso et al. (1997) using VLA data. To produce this plot, we have considered only our regions around the rim of Tycho, and we have adopted the mean of the expansion parameters over the azimuthal angles which correspond to those regions using the data in Table 2 of Reynoso et al. (1997). At $m>0.4$, the rolloff energy appears to increase with $m$, suggesting the least decelerated (fastest) shocks of Tycho are the most efficient at accelerating particles to high energies. The minimum rolloff energy is around $m=0.4$ where the SNR has reached its Sedov phase. At $m=0.25$, where the shock is encountering dense material, the SNR is slightly more efficient at accelerating particles, reaching half of the rolloff energy of the high- $m$ regions.

In Figure 12, we plot the rolloff energy $E_{\text {rolloff }}$ versus the post-shock density $n_{\mathrm{o}}$ (left panel) and versus the shock velocity $v_{\mathrm{s}}$ (right panel). Both of these quantities were derived by Williams et al. (2013) using Spitzer Space Telescope 24 and 70 $\mu \mathrm{m}$ data as well as the radio and X-ray proper motions of Reynoso et al. (1997) and Katsuda et al. (2010). We find that the regions of large $E_{\text {rolloff }}$ have the lowest post-shock densities (with $n_{\mathrm{o}} \lesssim 0.25 \mathrm{~cm}^{-3}$ ) and the largest shock velocities (with $v_{\mathrm{s}}$ $\gtrsim 3500 \mathrm{~km} \mathrm{~s}^{-1}$ ). The two outliers to these trends (those with $n_{0}>1 \mathrm{~cm}^{-3}, v_{\mathrm{s}}<2200 \mathrm{~km} \mathrm{~s}^{-1}$, and $E_{\text {rolloff }}>0.8 \mathrm{keV}$ ) correspond to regions \#12 and 21, where the shock is interacting with knot $g$.

We note that the conversion of proper motions to shock velocities requires an assumption about the distance to Tycho, which is still uncertain, with estimates in the literature ranging from 1.7 to $5.0 \mathrm{kpc}$ (e.g., Albinson et al. 1986; Schwarz et al. 1995; Völk et al. 2008; Hayato et al. 2010; Tian \& Leahy 2011; Slane et al. 2014). For the data plotted in Figure 12, Williams et al. (2013) employed a distance of $D=2.3 \mathrm{kpc}$, as suggested by Chevalier et al. (1980). The shock velocities scale linearly with the assumed distance, so the choice of distance will shift the points in the right panel of Figure 12 accordingly.

The relationship between $E_{\text {rolloff }}$ and $v_{\mathrm{s}}$ in Figure 12 can be used to examine the mechanism limiting the maximum energy of electrons $E_{\max }$ undergoing DSA. The timescale $t_{\mathrm{acc}}$ of the acceleration is set by the lesser of three relevant processes: the age of the SNR (i.e., the finite time the electrons have had to accelerate), the timescale of the radiative losses of the electrons, and the timescale for the particles to escape upstream of the shock. In each case, $E_{\max }$ (and consequently, $E_{\text {rolloff }}$ ) has different dependences on physical parameters (from Reynolds 2008, Equations (26)-(28)), specifically $v_{\mathrm{s}}$ :

$$
\begin{gathered}
E_{\max }(\text { age }) \propto v_{\mathrm{s}}^{2} t B\left(\eta R_{\mathcal{J}}\right)^{-1} \\
E_{\max }(\text { loss }) \propto v_{\mathrm{s}}\left(\eta R_{\mathcal{J}} B\right)^{-1 / 2} \\
E_{\max }(\text { esc }) \propto B \lambda_{\max } .
\end{gathered}
$$

In the above relations, $t$ is the age of the SNR, $\eta$ is the gyrofactor (where $\eta=1$ is Bohm diffusion and $\eta>1$ is "Bohm-like" diffusion), and $\lambda_{\max }$ is the maximum wavelength of MHD waves present to scatter electrons. $R_{\mathcal{J}}$ parametrizes the obliquity dependence of the acceleration: given that the shock obliquity angle $\theta_{\mathrm{Bn}}$ is the angle between the mean upstream 

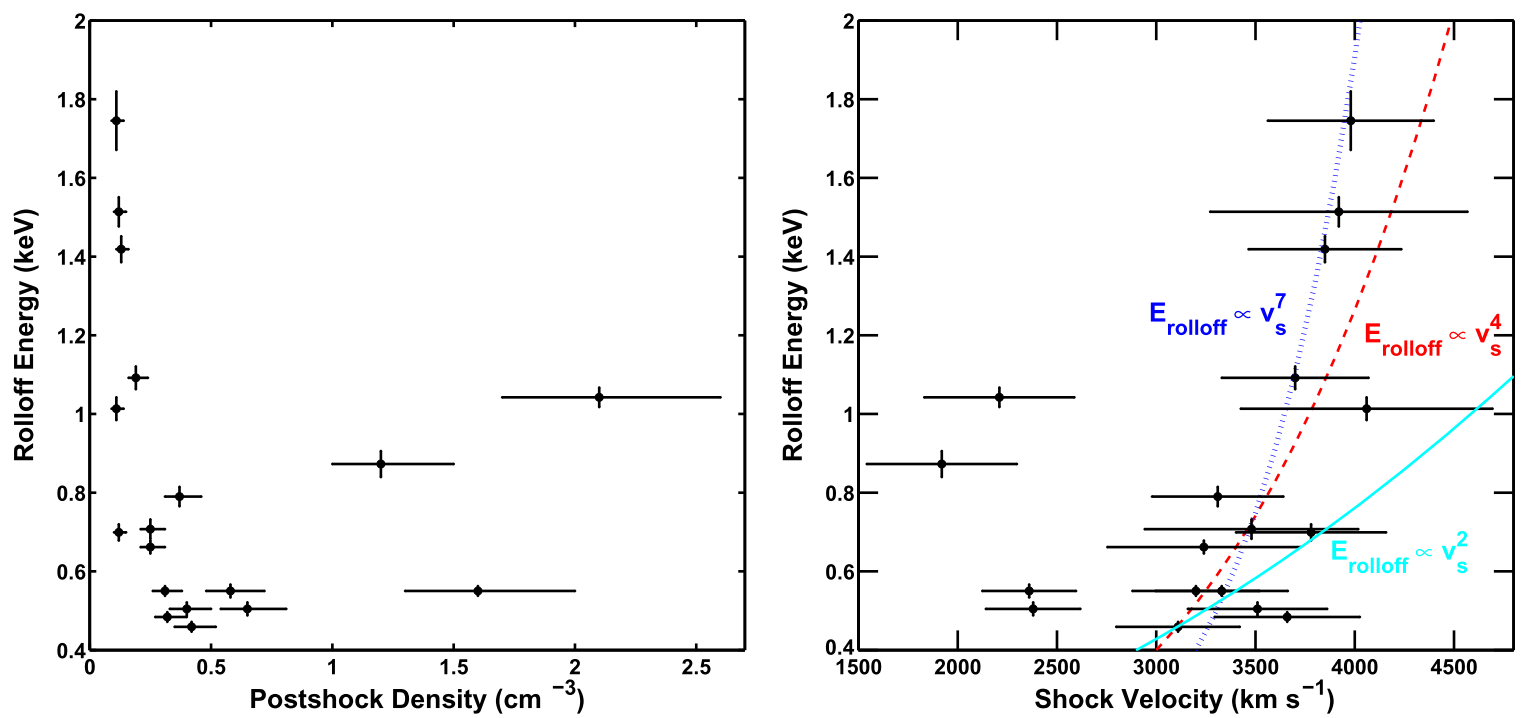

Figure 12. Rolloff energy $E_{\text {rolloff }}$ vs. post-shock density $n_{\mathrm{o}}$ (left) and shock velocity $v_{\mathrm{s}}$ (right) derived by Williams et al. (2013) adopting a distance of $D=2.3 \mathrm{kpc}$. The velocities scale linearly with the assumed distance, but the trend of $E_{\text {rolloff }}$ with $v_{\mathrm{s}}$ is independent of distance. Overplotted on the right panel are three curves that represent different dependences of $E_{\text {rolloff }}$ on shock velocity $v_{\mathrm{s}}: E_{\text {rolloff }} \propto v_{\mathrm{s}}^{2}$ (cyan solid line), $E_{\text {rolloff }} \propto v_{\mathrm{s}}^{4}$ (red dashed line), and $E_{\text {rolloff }} \propto v_{\mathrm{s}}^{7}$ (blue dotted line). The former two trends are those expected if the maximum energy of the electrons is limited by the age of the SNR or by the timescale of the radiative losses, respectively. The latter curve represents the loss-limited case if the B-field strength is not constant (and instead $B \propto v_{\mathrm{s}}$ ). The $E_{\text {rolloff }} \propto v_{\mathrm{s}}^{2}$ trend does not match the data, and the agelimited scenario is more consistent with our results. We note that the three $n_{0}>1 \mathrm{~cm}^{-3}$ points correspond to regions \#12, 10, and 21 (in order of increasing $n_{0}$ ). The two $v_{\mathrm{s}} \lesssim 2200 \mathrm{~km} \mathrm{~s}^{-1}$ points correspond also to regions \#12 and 21, which are located where the forward shock is interacting with the dense clump known as knot $g$ (Kamper \& van den Bergh 1978; Ghavamian et al. 2000).

B-field direction and the shock velocity, then the quantity $R_{\mathcal{J}}$ is defined as $R_{\mathcal{J}} \equiv t_{\mathrm{acc}}\left(\theta_{\mathrm{Bn}}\right) / t_{\mathrm{acc}}\left(\theta_{\mathrm{Bn}}=0\right)$. Since $E_{\text {rolloff }} \propto E_{\max }^{2} B$, the above relations can be rewritten in terms of $E_{\text {rolloff: }}$ :

$$
\begin{gathered}
E_{\text {rolloff }}(\text { age }) \propto v_{\mathrm{s}}^{4} t^{2} B^{3}\left(\eta R_{\mathcal{J}}\right)^{-2} \\
E_{\text {rolloff }}(\text { loss }) \propto v_{\mathrm{s}}^{2}\left(\eta R_{\mathcal{J}}\right)^{-1} \\
E_{\text {rolloff }}(\mathrm{esc}) \propto B^{3} \lambda_{\max }^{2} .
\end{gathered}
$$

In the right panel of Figure 12, we plot curves for $E_{\text {rolloff }} \propto v_{\mathrm{s}}^{2}$ (solid light blue line) and $E_{\text {rolloff }} \propto v_{\mathrm{s}}^{4}$ (dashed red line), for comparison. Additionally, since $v_{\mathrm{s}}=m R / t \propto m$ (where $R$ is the radius of the shock and $t$ is the age of the SNR), we have plotted the same curves on Figure 11 as well. The two dependencies of $E_{\text {rolloff }}$ on $v_{\mathrm{s}}$ reflect those of the loss-limited and age-limited relations above, assuming constant $B$ and $R_{\mathcal{J}}$. We find that the $\propto v_{\mathrm{s}}^{2}$ curve is too shallow to match the highvelocity $\left(v_{\mathrm{s}} \gtrsim 3500 \mathrm{~km} \mathrm{~s}^{-1}\right)$ points, and the $\propto v_{\mathrm{s}}^{4}$ curve is a better descriptor of the data. We note that if $B$ is not constant and is instead amplified by a constant fraction of the shock kinetic energy, then $B \propto v_{\mathrm{s}}$, and Equation (6) gives $E_{\text {rolloff }} \propto v_{\mathrm{s}}^{7}$. We have plotted this steeper curve in Figures 11 and 12 as well, and it adequately describes the data. Due to the relatively large error bars on the shock velocity $v_{\mathrm{s}}$, we are not able to distinguish which curve $\left(E_{\text {rolloff }} \propto v_{\mathrm{s}}^{4}\right.$ or $\left.E_{\text {rolloff }} \propto v_{\mathrm{s}}^{7}\right)$ is better statistically, but both are much more successful than the loss-limited case (with $E_{\text {rolloff }} \propto v_{\mathrm{s}}^{2}$, with no dependence on magnetic-field strength $B$ ) and the escape-limited case (with no $E_{\text {rolloff }}$ dependence on $v_{\mathrm{s}}$ ). Thus, our spatially resolved spectroscopic results are most consistent with the age-limited scenario, whereby the maximum electron energy $E_{\max }$ is determined by the finite time the electrons have had to accelerate. In this case, the accelerated electrons achieve the same maximum energy as the protons/ions because the electrons are not limited by their radiative losses.
However, previous studies of Tycho have argued that the SNR is likely loss-limited instead of age-limited (although see Reynolds \& Keohane 1999). In particular, Parizot et al. (2006) and Helder et al. (2012) calculate the synchrotron loss timescale and found it to be a few percent of the age of Tycho. Furthermore, recent hydrodynamical simulations by Slane et al. (2014) show that the loss-limited scenario is consistent with the observed broadband spectrum of Tycho. We note that these models were matched to the global spectra across Tycho as the gamma-ray emission is not resolved. Thus, local variations in the gamma-ray emission is possible, with the implication that the electrons and protons may achieve the same maximum energies in certain locations.

For the acceleration to be age limited, the synchrotron loss time $t_{\text {syn }}$ for X-ray emitting electrons must be larger than the remnant age, $t=442$ years for Tycho. As a consistency check, we estimate the magnetic field strength $B$ necessary for $t_{\mathrm{syn}}>t$ and compare it to that measured observationally. The synchrotron loss time $t_{\mathrm{syn}}$ is related to the $E_{\max }$ and $E_{\text {rolloff }}$ by (adapted from Vink 2012)

$$
\begin{aligned}
& t_{\mathrm{syn}}=12.5\left(\frac{E_{\mathrm{max}}}{100 \mathrm{TeV}}\right)^{-1}\left(\frac{B}{100 \mu \mathrm{G}}\right)^{-2} \text { year } \\
& t_{\mathrm{syn}}=104\left(\frac{E_{\text {rolloff }}}{1 \mathrm{keV}}\right)^{-1 / 2}\left(\frac{B}{100 \mu \mathrm{G}}\right)^{-3 / 2} \text { year. }
\end{aligned}
$$

Adopting the highest rolloff frequency from our spectral analysis, $\nu_{\text {rolloff }}=5.38 \times 10^{17} \mathrm{~Hz}$ (from region \#20), we derive $B=29 \mu \mathrm{G}$. This value is far below the measurements by Parizot et al. (2006) of $B=200 \mu \mathrm{G}$ based on the assumption that the X-ray filament widths are limited by the synchrotron loss time. However, it is possible that filament widths are governed instead by magnetic field damping (e.g., Pohl et al. 2005; Rettig \& Pohl 2012; Ressler et al. 2014), and 
this mechanism is the only possible one if the rims are equally thin at radio wavelengths, as is true in some regions of Tycho. Thus, the age-limited interpretation of our NuSTAR results would demand low magnetic field strengths and magnetic-field damping to be responsible for the X-ray rim morphology. However, a recent detailed analysis of Tycho (Tran et al. 2015) finds that even in damping models, magnetic field strengths $\gg$ $30 \mu \mathrm{G}$ are required to fit the radio and X-ray profiles of the thin rims.

Alternatively, the electrons are loss limited, and the apparent trend of $E_{\text {rolloff }}$ with $v_{\mathrm{s}}$ arises because of obliquity effects. In the scalings relating $E_{\text {rolloff }}$ to $v_{\mathrm{s}}$, we have assumed constant $R_{\mathcal{J}}$, yet the shock obliquity $\theta_{\mathrm{Bn}}$ is expected to change around the periphery of the SNR as the shock encounters a roughly uniform magnetic field in the environs of an SN Ia (Reynolds 1998). For example, changes in obliquity are thought to be the origin of the X-ray bright, synchrotron rims aligned in the northeast and southwest directions of SN 1006 (Rothenflug et al. 2004; Reynoso et al. 2013). The magnetic field is not as ordered in Tycho as in SN 1006 (see Figure 8 of Reynoso et al. 1997), and further work is necessary to assess the obliquity dependence of the particle acceleration in Tycho.

\section{CONCLUSIONS}

We have reported the large, $\sim 750 \mathrm{ks} N u S T A R$ observing program toward the historical SNR Tycho. Using these data, we produced narrow-band images over several energy bands to identify the locations of the hardest X-rays and to search for radioactive decay line emission from ${ }^{44} \mathrm{Ti}$. We find that the hardest $>10 \mathrm{keV} \mathrm{X}$-rays are concentrated to the southwest of Tycho, where recent Chandra observations have revealed high emissivity "stripes" associated with particles accelerated to the knee of the cosmic-ray spectrum. We do not find evidence of ${ }^{44} \mathrm{Ti}$, and we set limits on the presence of ${ }^{44} \mathrm{Ti}$, depending on the velocity and distribution of the metal. In order to be consistent with the reported Swift/BAT detection, the Ti must be expanding at moderate-to-high velocities and/or distributed over the majority of the SNR. Our spatially resolved spectroscopic analyses of sixty-six regions showed that the highest energy electrons are accelerated by the fastest shocks and in the lowest density regions of the SNR. We find a steep dependence of the rolloff frequency with shock velocity that is consistent with the maximum energy of accelerated electrons being limited by the age of the SNR rather than by synchrotron losses, contrary to previous results obtained for Tycho. One way to reconcile these discrepant findings is through shock obliquity effects, and future observational work is necessary to explore the role of obliquity in the particle acceleration process.

We acknowledge helpful discussions with Drs. Marco Miceli, Lorenzo Sironi, and Patrick Slane. L.A.L. received support for this work from NASA through Hubble Fellowship grant number HST-HF2-51342.001 awarded by the Space Telescope Science Institute, which is operated by the Association of Universities for Research in Astronomy, Inc., for NASA, under contract NAS 5-26555. Additionally, the work was supported under NASA contract NNG08FD60C and made use of data from the NuSTAR mission, a project led by the California Institute of Technology, managed by the Jet Propulsion Laboratory, and funded by NASA. We thank the NuSTAR Operations, Software, and Calibration teams for support with the execution and analysis of these observations. This research made use of the NUSTAR Data Analysis Software (NuSTARDAS), jointly developed by the ASI Science Data Center (ASDC, Italy) and the California Institute of Technology (USA).

\section{Facilities: NuSTAR, CXO, VLA.}

\section{REFERENCES}

Acciari, V. A., Aliu, E., Arlen, T., et al. 2011, ApJL, 730, L20

Ahmad, I., Greene, J. P., Moore, E. F., et al. 2006, PhRvC, 74, 065803

Albinson, J. S., Tuffs, R. J., Swinbank, E., \& Gull, S. F. 1986, MNRAS, 219,427

Atoyan, A., \& Dermer, C. D. 2012, ApJL, 749, L26

Badenes, C., Borkowski, K. J., Hughes, J. P., Hwang, U., \& Bravo, E. 2006, ApJ, 645, 1373

Bamba, A., Yamazaki, R., Yoshida, T., Terasawa, T., \& Koyama, K. 2005, ApJ, 621, 793

Berezhko, E. G., Ksenofontov, L. T., \& Völk, H. J. 2013, ApJ, 763, 14

Blondin, J. M., Wright, E. B., Borkowski, K. J., \& Reynolds, S. P. 1998, ApJ, 500,342

Boggs, S. E., Harrison, F. A., Miyasaka, H., et al. 2015, Sci, 348, 670 Borkowski, K. J., Reynolds, S. P., Green, D. A., et al. 2010, ApJ, 724, 161 Bykov, A. M., Ellison, D. C., Osipov, S. M., Pavlov, G. G., \& Uvarov, Y. A. 2011, ApJL, 735, L40

Chevalier, R. A. 1982a, ApJL, 259, L85

Chevalier, R. A. 1982b, ApJ, 258, 790

Chevalier, R. A., Kirshner, R. P., \& Raymond, J. C. 1980, ApJ, 235, 186

Cioffi, D. F., McKee, C. F., \& Bertschinger, E. 1988, ApJ, 334, 252

Decourchelle, A., Sauvageot, J. L., Audard, M., et al. 2001, A\&A, 365, L218

Dupraz, C., Bloemen, H., Bennett, K., et al. 1997, A\&A, 324, 683

Eriksen, K. A., Hughes, J. P., Badenes, C., et al. 2011, ApJL, 728, L28

Fink, M., Röpke, F. K., Hillebrandt, W., et al. 2010, A\&A, 514, A53

Ghavamian, P., Raymond, J., Hartigan, P., \& Blair, W. P. 2000, ApJ, 535, 266

Giordano, F., Naumann-Godo, M., Ballet, J., et al. 2012, ApJL, 744, L2

Grebenev, S. A., Lutovinov, A. A., Tsygankov, S. S., \& Winkler, C. 2012, Natur, 490, 373

Grefenstette, B. W., Harrison, F. A., Boggs, S. E., et al. 2014, Natur, 506, 339 Grefenstette, B. W., Reynolds, S. P., Harrison, F. A., et al. 2015, ApJ, 802, 15 Harrison, F. A., Craig, W. W., Christensen, F. E., et al. 2013, ApJ, 770, 103 Hayato, A., Yamaguchi, H., Tamagawa, T., et al. 2010, ApJ, 725, 894

Helder, E. A., Vink, J., Bykov, A. M., et al. 2012, SSRv, 173, 369

Hwang, U., Decourchelle, A., Holt, S. S., \& Petre, R. 2002, ApJ, 581, 1101 Iwamoto, K., Brachwitz, F., Nomoto, K., et al. 1999, ApJS, 125, 439 Iyudin, A. F., Diehl, R., Bloemen, H., et al. 1994, A\&A, 284, L1 Iyudin, A. F., Schönfelder, V., Bennett, K., et al. 1999, ApL\&C, 38, 383

Kamper, K. W., \& van den Bergh, S. 1978, ApJ, 224, 851

Katsuda, S., Petre, R., Hughes, J. P., et al. 2010, ApJ, 709, 1387

Kothes, R., Fedotov, K., Foster, T. J., \& Uyanıker, B. 2006, A\&A, 457, 1081

Krause, O., Tanaka, M., Usuda, T., et al. 2008, Natur, 456, 617

Lee, J.-J., Koo, B.-C., \& Tatematsu, K. 2004, ApJL, 605, L113

Maeda, K., Röpke, F. K., Fink, M., et al. 2010, ApJ, 712, 624

Magkotsios, G., Timmes, F. X., Hungerford, A. L., et al. 2010, ApJS, 191, 66

Miceli, M., Sciortino, S., Troja, E., \& Orlando, S. 2015, ApJ, 805, 120

Moll, R., \& Woosley, S. E. 2013, ApJ, 774, 137

Parizot, E., Marcowith, A., Ballet, J., \& Gallant, Y. A. 2006, A\&A, 453, 387 Pohl, M., Yan, H., \& Lazarian, A. 2005, ApJL, 626, L101

Pravdo, S. H., \& Smith, B. W. 1979, ApJL, 234, L195

Renaud, M., Vink, J., Decourchelle, A., et al. 2006a, NewAR, 50, 540 Renaud, M., Vink, J., Decourchelle, A., et al. 2006b, ApJL, 647, L41 Ressler, S. M., Katsuda, S., Reynolds, S. P., et al. 2014, ApJ, 790, 85 Rest, A., Welch, D. L., Suntzeff, N. B., et al. 2008, ApJL, 681, L81

Rettig, R., \& Pohl, M. 2012, A\&A, 545, A47

Reynolds, S. P. 1998, ApJ, 493, 375

Reynolds, S. P. 2008, ARA\&A, 46, 89

Reynolds, S. P., \& Ellison, D. C. 1992, ApJL, 399, L75

Reynolds, S. P., \& Keohane, J. W. 1999, ApJ, 525, 368

Reynoso, E. M., Hughes, J. P., \& Moffett, D. A. 2013, AJ, 145, 104

Reynoso, E. M., Moffett, D. A., Goss, W. M., et al. 1997, ApJ, 491, 816

Reynoso, E. M., Velázquez, P. F., Dubner, G. M., \& Goss, W. M. 1999, AJ, 117,1827

Rothenflug, R., Ballet, J., Dubner, G., et al. 2004, A\&A, 425, 121

Schwarz, U. J., Goss, W. M., Kalberla, P. M., \& Benaglia, P. 1995, A\&A, 299, 193

Slane, P., Lee, S.-H., Ellison, D. C., et al. 2014, ApJ, 783, 33 
Stephenson, F. R., \& Green, D. A. 2002, in Historical Supernovae and their Remnants, Vol. 5, ed. F. Richard Stephenson \& David A. Green (Oxford: Clarendon), 5

Tian, W. W., \& Leahy, D. A. 2011, ApJL, 729, L15

Timmes, F. X., Woosley, S. E., Hartmann, D. H., \& Hoffman, R. D. 1996, ApJ, 464,332

Tran, A., Williams, B. J., Petre, R., Ressler, S. M., \& Reynolds, S. P. 2015, arXiv: 1509.00877

Troja, E., Segreto, A., La Parola, V., et al. 2014, ApJL, 797, L6

Vink, J. 2012, A\&ARv, 20, 49

Vink, J., Laming, J. M., Kaastra, J. S., et al. 2001, ApJL, 560, L79
Völk, H. J., Berezhko, E. G., \& Ksenofontov, L. T. 2008, A\&A, 483, 529

Wang, W., \& Li, Z. 2014, ApJ, 789, 123

Warren, J. S., Hughes, J. P., Badenes, C., et al. 2005, ApJ, 634, 376

Wik, D. R., Hornstrup, A., Molendi, S., et al. 2014, ApJ, 792, 48

Williams, B. J., Borkowski, K. J., Ghavamian, P., et al. 2013, ApJ, 770, 129

Woosley, S. E., Heger, A., \& Weaver, T. A. 2002, RvMP, 74, 1015

Woosley, S. E., \& Kasen, D. 2011, ApJ, 734, 38

Yamaguchi, H., Eriksen, K. A., Badenes, C., et al. 2014, ApJ, 780, 136

Zoglauer, A., Reynolds, S. P., An, H., et al. 2015, ApJ, 798, 98 